\title{
Statistical Entropy Analysis to Evaluate Cascading Use of Wood ${ }^{+}$
}

\author{
Kranti Navare 1,2, Karl Vrancken ${ }^{2,3}$ and Karel Van Acker 1,4 \\ 1 Department of Materials Engineering, KU Leuven, Kasteelpark Arenberg 44, Leuven, Belgium \\ 2 VITO, Sustainable Materials, Boeretang 200, Mol, Belgium \\ 3 Department of Bio-engineering, University of Antwerp, Groenenborgerlaan 171, Antwerpen, Belgium \\ 4 Research Centre for Economics and Corporate Sustainability, KU Leuven, Warmoesberg 26, Brussels, \\ Belgium \\ + Presented at the Entropy 2021: The Scientific Tool of the 21st Century, 5-7 May 2021; Available online: \\ https://sciforum.net/conference/Entropy2021/.
}

Published: 5 May 2021

Biological materials are biodegradable in nature. Consequently, it is harder to preserve their material value. Recycling these materials to its original form is difficult. Hence the material is cascaded down in its application. Cascading use implies a system in which biomass progresses through a series of uses before finally being burned to recover energy. The aim is to preserve the material quality and prioritize the use based on the maximum added value that can potentially be generated from it. For instance, sawn wood should preferably be used for building, furniture and other products with a long life span, while bioenergy should be derived from the use of wood residues. However, identifying the best valorisation routes requires appropriate measurement and monitoring tools to quantify the degree of cascading, which is still lacking.

Statistical entropy analysis (SEA) has been put forward as a method to quantify resource quality. This could be a powerful tool to assess cascading use. SEA measures the concentration of material along its life cycle and determines the efficiency of a system based on its ability to concentrate or dilute a substance. The concentration of material has been proposed as a proxy for quality; the higher the concentration of a material, the higher is its availability, and hence the higher would be its recoverability and recyclability. However, in the case of biological material, in particular wood, along with concentration product-size dictates the quality. Product-size is not considered in the traditional SEA methodologies, which limits its applicability to the biological material. The goal of this study is to adapt the SEA methodology to incorporate physical dimensionality. The adapted method, demonstrated by comparing different wood cascading scenarios in Flanders (Belgium), reveals valuable information about key drivers of quality loss in the value chain and identifies hotspots for improvement.

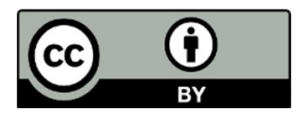

(C) 2021 by the authors. Licensee MDPI, Basel, Switzerland. This article is an open access article distributed under the terms and conditions of the Creative Commons Attribution (CC BY) license (http://creativecommons.org/licenses/by/4.0/). 